\section{Considerations on Double Exponential-Based Cubatures for the Computation of Weakly Singular Galerkin Inner Products}

\author{
Athanasios G. Polimeridis, Ioannis D. Koufogiannis, \\ Michael Mattes, and Juan R. Mosig
}

\begin{abstract}
Highly accurate and efficient cubatures based on the double exponential quadrature rules are presented for the computation of weakly singular integrals arising in Galerkin mixed potential integral equation formulations. Due to their unique ability to handle non-smooth kernels, the proposed integration schemes can safely replace (in a "plug-n-play" sense) the traditional Gauss-Legendre rules in the existing singularity cancellation and singularity subtraction methods. Numerical examples using RWG basis functions confirm the excellent performance of the proposed method.
\end{abstract}

Index Terms-Double exponential quadrature, method of moments (MoM), mixed potential integral equation (MPIE), RWG basis functions, weakly singular integrals.

\section{INTRODUCTION}

Mixed potential integral equation (MPIE) formulations, together with a Galerkin discretization, stand as one of the most powerful variants of the generic surface integral equations (SIEs) for the solution of electromagnetic (EM) scattering problems, especially in planar stratified geometries [1]. One of the key features of MPIE formulations is that they involve only the potential forms of Green's functions. Even in this case, though, the arising 4-D weakly singular (improper Riemann integrable) integrals call for special treatment in order to be accurately and efficiently evaluated.

Traditionally, weakly singular multidimensional integrals are tackled by the so-called singularity subtraction and singularity cancellation methods. Although each one of these methods utilizes different concepts for the regularization of the singular kernels, they both deal with the original 4-D integrals by considering separately the outer (observation) and the inner (source or potential) 2-D integrals. In that case, even if the potential integral is accurately evaluated to machine precision, the overall accuracy for the original 4-D integral is limited due to the non-sufficiently smooth behavior of the potential as a function of the outer integration's arguments. In other words, even if we have at hand complete analytical expressions for the associated inner potential integrals, the unbounded nature of their higher derivatives restrain typical interpolatory cubatures, like the standard Gauss-Legendre (GL), from reaching the desired accuracy in a reasonable computation time. Interested readers could consult some of the recent publications [2]-[4] for a more detailed overview of previous work relevant to the evaluation of weakly singular integrals in computational electromagnetics.

The focal point of this study lies on the never ending quest for accurate and efficient evaluation of the outer 2-D integrals, provided that the inner potential integral is computed to machine precision by means

Manuscript received March 01, 2011; revised June 22, 2011; accepted October 10, 2011. Date of publication March 02, 2012; date of current version May 01,2012 . This work was supported by the COST Actions IC0803-RFCSET and IC0603-ASSIST.

The authors are with the Laboratory of Electromagnetics and Acoustics (LEMA), Ecole Polytechnique Fédérale de Lausanne (EPFL), CH-1015 Lausanne, Switzerland (e-mail: athanasios.polymeridis@epfl.ch; ioannis.koufogiannis@epfl.ch; michael.mattes@epfl.ch; juan.mosig@epfl.ch).

Digital Object Identifier 10.1109/TAP.2012.2189708 of the aforementioned methods, which are readily available in the literature. More specifically, herein, we elaborate further on the cubatures that were recently presented in [5], by exploiting the prospects of their main building block, i.e., the double exponential (DE) transformation [6]. It is the unique ability of this type of quadrature rules to handle integrands with end-point singularities (see also the IMT rule [7]), that designates them perfect candidates for the generalized Cartesian product rules under investigation.

As detailed in our previous work [5], DE-based cubatures clearly outperform GL integration schemes, leading even to machine precision results for the aforesaid 4-D weakly singular and near-singular integrals. The only drawback of the novel cubatures stems from the slow convergence when it comes to lower accuracy, i.e., GL rules are more efficient for relative errors of less significant digits. In next, we resort to the "reverse engineering" rationale in order to overcome this shortcoming. The performance of the final cubatures, which are based on slightly modified DE transformations, allows us to safely replace the traditional 2-D cubatures (mainly GL) in the existing singularity cancellation and singularity subtraction methods and fill up the associated method of moments (MoM) impedance matrix with highly accurate entries in reasonable run times.

\section{Problem Statement}

The evaluation of the Galerkin MoM matrix elements in MPIE, using div-conforming (linear or of higher order) basis $\left(\mathbf{f}_{m}\right)$ and testing $\left(\mathbf{f}_{n}\right)$ functions, calls for the computation of the following 4-D integrals:

$$
\begin{aligned}
I_{1} & :=\int_{E_{P}} \nabla \cdot \mathbf{f}_{m}(\mathbf{r}) \int_{E_{Q}} G\left(\mathbf{r}, \mathbf{r}^{\prime}\right) \nabla^{\prime} \cdot \mathbf{f}_{n}\left(\mathbf{r}^{\prime}\right) d S^{\prime} d S \\
I_{2} & :=\int_{E_{P}} \mathbf{f}_{m}(\mathbf{r}) \cdot \int_{E_{Q}} G\left(\mathbf{r}, \mathbf{r}^{\prime}\right) \mathbf{f}_{n}\left(\mathbf{r}^{\prime}\right) d S^{\prime} d S
\end{aligned}
$$

which incorporate the free-space Green's function

$$
G\left(\mathbf{r}, \mathbf{r}^{\prime}\right)=G(R)=\frac{e^{-j k R}}{R}
$$

with $R=\left|\mathbf{r}-\mathbf{r}^{\prime}\right|$ being the distance function and $k=\omega \sqrt{\varepsilon \mu}$ the wavenumber. Traditionally, $E_{P}$ and $E_{Q}$ correspond to the supports of the associated testing and basis functions, respectively, but systematic object-oriented programming dictates an "element-by-element" approach, where $E_{P}$ and $E_{Q}$ correspond to the elements of the mesh (triangles, quadrilaterals etc.). The weakly singular cases for the integrals above arise when the observation and source elements $E_{P}$ and $E_{Q}$, respectively, coincide or share some common points.

The basic idea in singularity cancellation and singularity subtraction methods is to regularize appropriately the singular kernel in the inner potential integrals. Then, the bounded outer 2-D integral can be calculated with standard GL cubatures. But, as it was clearly demonstrated in our previous work [5], the accuracy and efficiency of those methods can be substantially improved upon utilizing more sophisticated quadrature rules, i.e., DE rules.

In the following, we elaborate further on the efficiency of these DE-based generalized Cartesian product rules. Note also that although we confine ourselves in this work to the evaluation of weakly singular integrals of RWG basis functions [8] over triangular elements, by means of the singularity cancellation method, the proposed integration schemes are really general-purpose and retain their applicability in numerous other cases (e.g., weakly singular integrals over quadrangular domains [9]). 


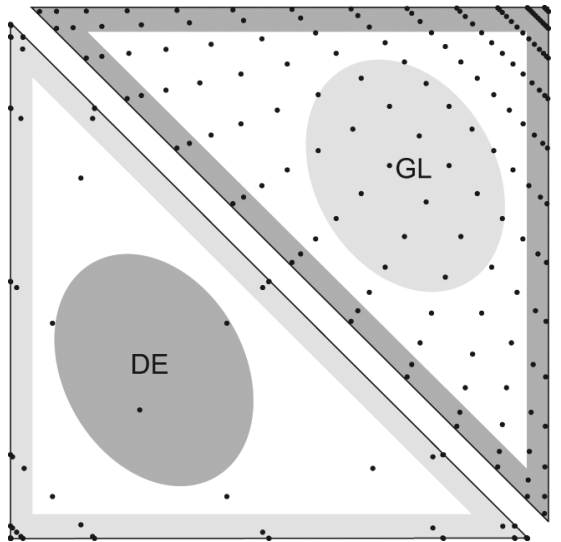

Fig. 1. Distribution of integration points for DE and GL cubatures over triangles. The same number of integration points is used for both but they are strongly concentrated in the edges for the DE case.

\section{DE-BASEd CubATURES}

The generalized 2-D Cartesian products rules introduced in [5] are based on the original DE quadrature rule

$$
\mathrm{I}_{h}^{(N)} f=h \sum_{k=-n}^{n} f(\phi(t)) \phi^{\prime}(t)
$$

where

$$
x=\phi(t)=\tanh \left(\frac{\pi}{2} \sinh (t)\right)
$$

and

$$
\begin{aligned}
\phi^{\prime}(t) & =\frac{\frac{\pi}{2} \cosh (t)}{\cosh ^{2}\left(\frac{\pi}{2} \sinh (t)\right)} \\
& \approx O\left(\exp \left(-\frac{\pi}{2} \exp (|t|)\right)\right),
\end{aligned}
$$

as $|t| \rightarrow \infty$. Note also that $\mathrm{I}_{h}^{(N)}$ stands for the trapezoidal formula with mesh size equal to $h$ and a total number of function evaluations equal to $N$. It is the unique ability of the DE transformation to handle functions with endpoint singularities that lead to unmatched accuracy for the evaluation of the outer 2-D integrals, in which case the kernel has infinite derivatives along the edges of the elements. The results presented in [5] fully justify such a statement. The only question (and motivation for this study) that arises naturally from examining the behavior of the DE-based cubatures concerns its overall efficiency, especially for lower accuracy.

By a simple inspection of the integration points' distribution for DE and GL cubatures (using for both the same number of integration points, although hard to visualize it due to limited resolution) over triangles, as depicted in Fig. 1, one can easily identify the source of the "problem": although DE cubature accumulates a sufficient number of points in the neighborhood of the edges (light grey region), where the kernel has unbounded derivatives, the region where the kernel is smooth is under-sampled compared to the GL cubature. Of course GL integration points cannot approach arbitrary close to the edges and extract useful information from the non-smooth behavior of the integrand, hence, the maximum accuracy is critically stagnated.

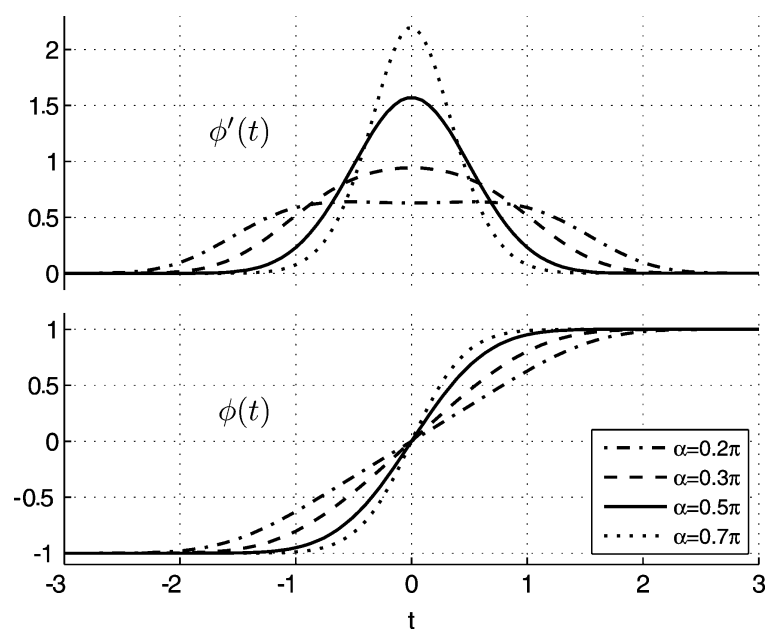

Fig. 2. DE transformation showing the influence of the new parameter $\alpha$.

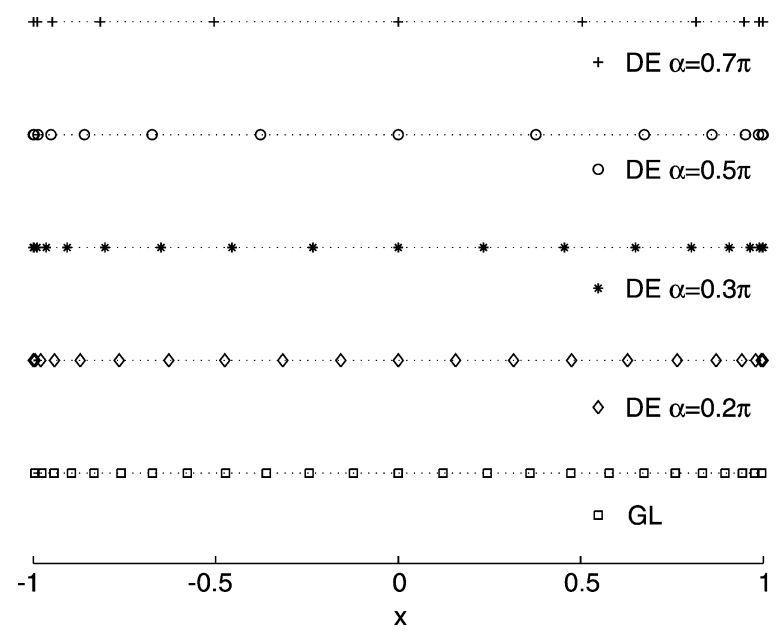

Fig. 3. Distribution of abscissas in the DE quadrature rule for different values of $\alpha$ compared with the GL quadrature.

\section{EFFICIENT DE CUBATURES}

In point of fact, the efficiency of the recently developed DE-based generalized Cartesian product rules can be improved if the density of the associated integration points in the area of the integration domain increases. This, in turn, calls for an appropriate modification of the original DE transformation (5). Indeed, the constant parameter $\pi / 2$ was originally introduced in [6] only to ease the error analysis. Moreover, in a related work [10] it was clearly stated that "other values of the parameter will not seriously impair the efficiency of the formula". Hence, we can modify it appropriately in order to improve (densify) the distribution of the integration points, without affecting the key feature of the quadrature rule, i.e., the double exponential decay of the associated weights $\phi^{\prime}(t)$.

The DE transformation with the free parameter $\alpha$ is given by

$$
\begin{aligned}
x & =\phi(t)=\tanh (\alpha \sinh (t)), \\
\phi^{\prime}(t) & =\frac{\alpha \cosh (t)}{\cosh ^{2}(\alpha \sinh (t))}
\end{aligned}
$$

with the corresponding behavior and integration points presented in Figs. 2 and 3, respectively. In addition, the exact number of integration points, in terms of the level (as explained in [5], [11]), for the modified DE quadrature rule are provided in Table I. 
TABLE I

NUMBER OF INTEGRATION POINTS $(N)$ FOR THE 1-D DE RULE IN TERMS OF THE ASSOCIATED LEVEL $M$

\begin{tabular}{c|cccccc}
\hline$N$ & 0 & 1 & 2 & 3 & 4 & 5 \\
\hline \hline$\alpha=0.2 \pi$ & 9 & 17 & 33 & 65 & 131 & 261 \\
\hline$\alpha=0.3 \pi$ & 7 & 15 & 29 & 59 & 117 & 235 \\
\hline$\alpha=0.5 \pi$ & 7 & 13 & 25 & 51 & 101 & 203 \\
\hline$\alpha=0.7 \pi$ & 5 & 11 & 23 & 45 & 91 & 181 \\
\hline
\end{tabular}

Evidently, a choice for the parameter $\alpha$ larger than the original value $\pi / 2$ would lead to even more sparse distribution of points in the area of interest, as shown in Fig. 3. On the other hand, for much smaller values of $\alpha$ the number of integration points close to the problematic endpoint is severely decreased and the DE quadrature is not able anymore to achieve high accuracy. Therefore, the optimal choice should be found somewhere in between and of course it would be ideal if this value was independent from the various geometrical (triangles' shapes) and algebraic (types of basis functions) characteristics of the weakly singular integrals under study. In fact, we can state here that this is roughly the case, as elucidated by the extensive numerical experiments that were undertaken. The "universal" optimal (in terms of efficiency) value of $\alpha$ was found to be close to $0.3 \pi$, instead of $0.5 \pi$ for the original version of DE.

\section{NUMERICAL RESULTS AND DISCUSSION}

Herein, we present some numerical results for typical 4-D weakly singular integrals, arising in Galerkin MPIE formulations. More specifically, the self-term cases

$$
\begin{aligned}
I_{\text {benchmark\#1 }} & =\int_{T_{1}} \int_{T_{1}} G\left(\mathbf{r}, \mathbf{r}^{\prime}\right) d S^{\prime} d S \\
I_{\text {benchmark\#2 }} & =\int_{T_{2}} \int_{T_{2}} G\left(\mathbf{r}, \mathbf{r}^{\prime}\right) d S^{\prime} d S
\end{aligned}
$$

and the edge adjacent cases

$$
\begin{aligned}
I_{\text {benchmark\#3 }} & =\int_{T_{3}} \int_{T_{2}} G\left(\mathbf{r}, \mathbf{r}^{\prime}\right) d S^{\prime} d S \\
I_{\text {benchmark\#4 }} & =\int_{T_{4}} \zeta_{1} \cdot \int_{T_{2}} G\left(\mathbf{r}, \mathbf{r}^{\prime}\right) \zeta_{1}^{\prime} d S^{\prime} d S
\end{aligned}
$$

are considered, where the wavelength is equal to $\lambda=1[\mathrm{~m}]$ and the triangles as well as the associated vertices are defined as follows:

$$
\begin{array}{ll}
T_{1}:\left\{\mathbf{r}_{1}, \mathbf{r}_{2}, \mathbf{r}_{3}\right\} ; & T_{2}:\left\{\mathbf{r}_{1}, \mathbf{r}_{2}, \mathbf{r}_{4}\right\} \\
T_{3}:\left\{\mathbf{r}_{1}, \mathbf{r}_{2}, \mathbf{r}_{5}\right\} ; & T_{4}:\left\{\mathbf{r}_{1}, \mathbf{r}_{2}, \mathbf{r}_{6}\right\}
\end{array}
$$

and

$$
\begin{array}{ll}
\mathbf{r}_{1}=[0,0,0] ; & \mathbf{r}_{2}=[0.1,0,0] \\
\mathbf{r}_{3}=[0,0.1,0] ; & \mathbf{r}_{4}=[-0.05,0.087,0] \\
\mathbf{r}_{5}=[-0.05,-0.087,0] ; & \mathbf{r}_{6}=[0,-0.1,0]
\end{array}
$$

respectively. Also, $\zeta_{1}$ and $\zeta_{1}^{\prime}$ correspond to the simplex coordinates of the associated vertices and the last integral appear in the vector potential part, when RWG basis are used [8].

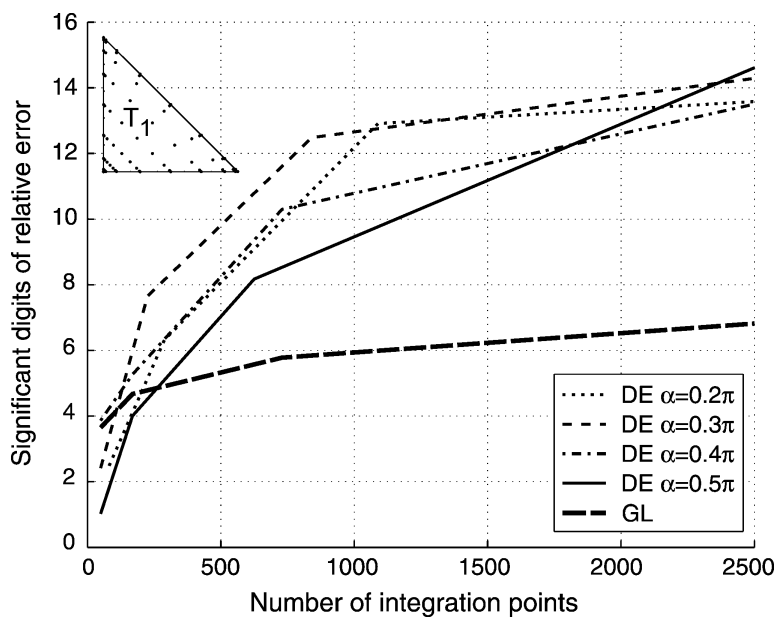

Fig. 4. Relative error in calculating the real part of the 4-D weakly singular integral (9), $I_{\text {benchmark\#1 }}$ (self-term).

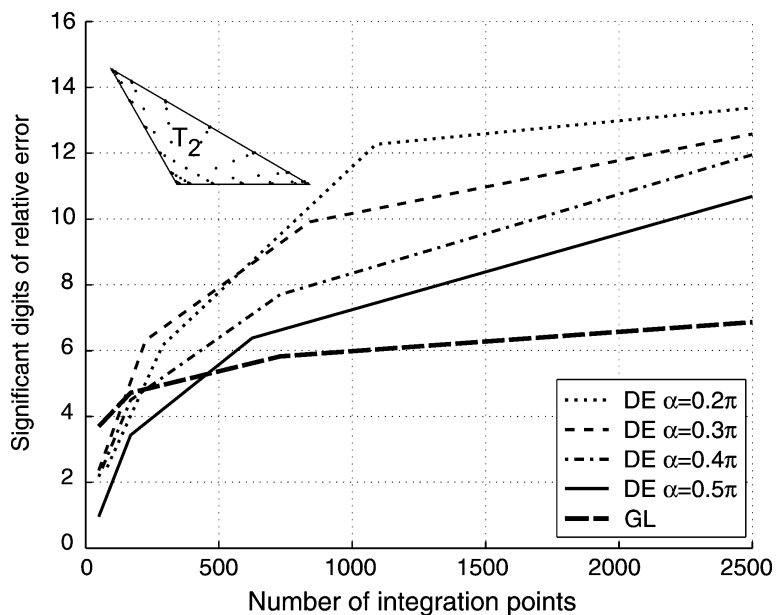

Fig. 5. Relative error in calculating the real part of the 4-D weakly singular integral (10), $I_{\text {benchmark\#2 }}$ (self-term).

The reference solution for all cases is obtained by means of the direct evaluation method [4], [12]. Note also that the focal point of this study is the performance of the 2-D cubatures for the outer integration, therefore, the inner 2-D integral is computed to machine precision with the help of the singularity cancellation scheme proposed by Khayat and Wilton [2]. In Figs. 4-7, the relative error in calculating the real part (singular portion) of the 4-D weakly singular integrals (9)-(12), respectively, is shown.

The overall comparison of the achieved accuracy in terms of the total number of integration points for the outer 2-D integral between the GL cubatures and the different variants of the DE cubatures fully justifies the aim of the presented study. More specifically, beginning with the first benchmark problem, i.e., the (scalar potential) self-term for an orthogonal isosceles triangle, one can easily identify the superior performance of the proposed optimal DE cubature against both GL and the different variants of DE cubatures. Even when efficiency is of first priority, the new DE cubature leads to reasonable high accuracy, yet without compromising the achievable maximum accuracy. Next, we have chosen two examples for the edge adjacent case where the source triangle is highly distorted (bad quality factor) and the observation (outer) triangle is either distorted (Fig. 6) or canonical (Fig. 7). It is worth mentioning, that also some other values for the parameter $\alpha$ 


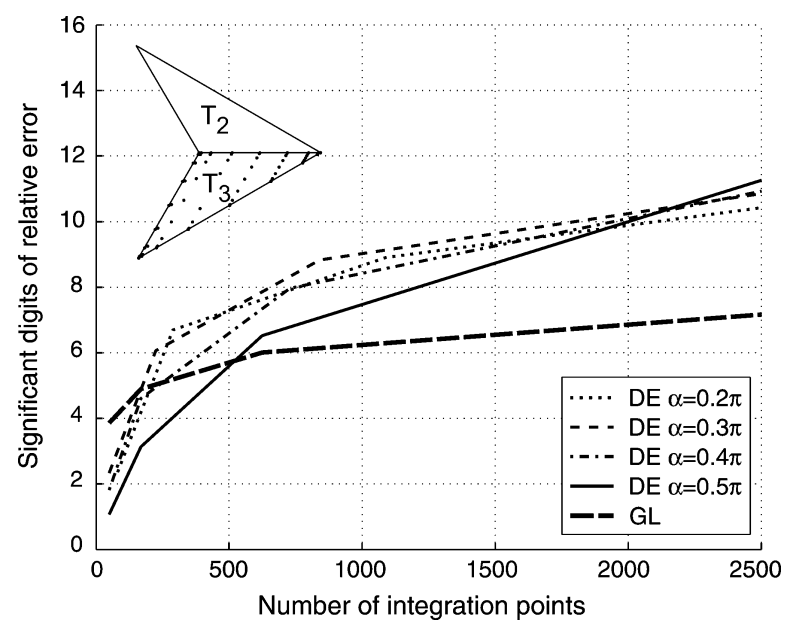

Fig. 6. Relative error in calculating the real part of the 4-D weakly singular integral (11), $I_{\text {benchmark\#3 }}$ (edge adjacent case).

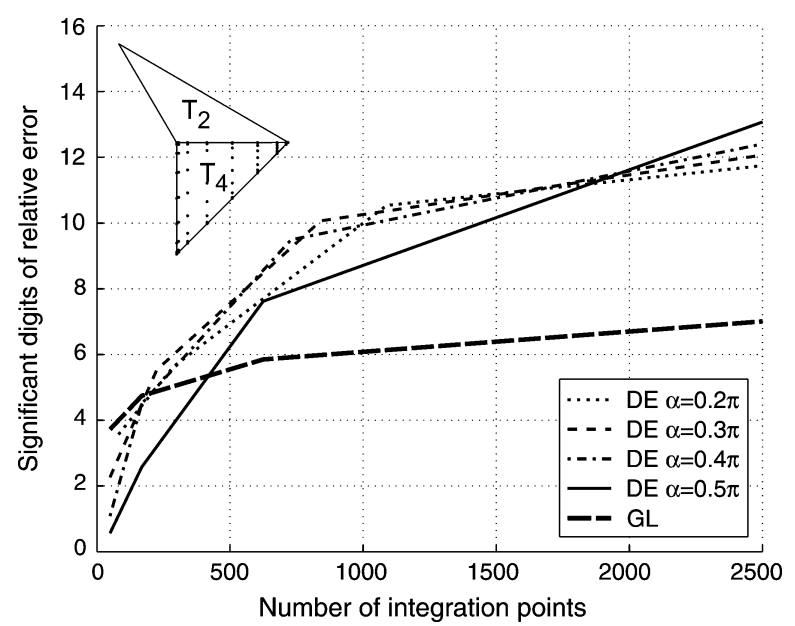

Fig. 7. Relative error in calculating the real part of the 4-D weakly singular integral (12), $I_{\text {benchmark\#4 }}$ (edge adjacent case).

close to the chosen value of $0.3 \pi$ can achieve optimum performance for some range of quality factors and number of sampling points, e.g., $\alpha=0.2 \pi$ for the distorted triangle of Fig. 5 .

In view of the presented results, no risks are taken by saying that the proposed DE cubatures can safely replace the various interpolatory integration schemes, readily available in the literature, in computing the 4-D weakly singular integrals together with the so-called singularity cancellation and singularity subtraction methods. Especially, incorporating DE cubatures into singularity cancellation schemes not only preserves object-oriented paradigms but can lead, for the first time to the best of our knowledge, to machine precision accuracy in reasonable run times. At this point, it is of paramount importance to stress the necessity for highly accurate computation of the impedance matrix entries in MoM, for the round-off error becomes more pronounced in the solution of linear systems with million unknowns that arise in the modern EM modelisation challenges. Indeed, machine precision computation can not be considered anymore blue sky research, but rather mainstream [3], [13].

Finally, it is worth mentioning that the proposed DE cubatures are expected to unveil their strong potential in much more challenging cases involving gradients of the Green's function, like the strongly singular integrals arising in other types of surface integral equations. Of course, this could be demonstrated quite easily provided that we had at hand a robust method for the accurate evaluation of strongly singular 2-D potential integrals, but unfortunately this is not the case today.

\section{CONCLUSION}

Efficient double exponential-based generalized Cartesian product rules are presented for the computation of 4-D weakly singular integrals, arising in Galerkin mixed potential integral equation formulations. The novel integration schemes are based on a smart modification of the double exponential transformation, in order to improve the distribution of the associated integration points. Finally, the proposed cubatures can be safely incorporated into singularity cancellation and singularity subtraction methods and improve substantially their overall accuracy in reasonable run times. In the future, even more challenging cases, like the strongly singular integrals appearing in other surface integral equation formulations, could be tackled with the very same rationale.

\section{REFERENCES}

[1] J. R. Mosig, R. C. Hall, and F. E. Gardiol, "Numerical analysis of microstrip patch antennas," in Handbook of Microstrip Antennas, James and Hall, Eds. London: IEE-Peter Peregrinus, 1989.

[2] M. A. Khayat and D. R. Wilton, "Numerical evaluation of singular and near-singular potential integrals," IEEE Trans. Antennas Propag., vol. 53, no. 10, pp. 3180-3190, Oct. 2005.

[3] R. D. Graglia and G. Lombardi, "Machine precision evaluation of singular and nearly singular potential integrals by use of Gauss quadrature formulas for rational functions," IEEE Trans. Antennas Propag., vol. 56, no. 4, pp. 981-998, Apr. 2008.

[4] A. G. Polimeridis and T. V. Yioultsis, "On the direct evaluation of weakly singular integrals in Galerkin mixed potential integral equation formulations," IEEE Trans. Antennas Propag., vol. 56, no. 9, pp. 3011-3019, Sep. 2008.

[5] A. G. Polimeridis and J. R. Mosig, "Evaluation of weakly singular integrals via generalized Cartesian product rules based on the double exponential formula," IEEE Trans. Antennas Propag., vol. 58, no. 6, pp. 1980-1988, Jun. 2010.

[6] H. Takahasi and M. Mori, "Double exponential formulas for numerical integration," Publ. RIMS, Kyoto Univ., no. 9, pp. 721-741, 1974.

[7] M. Iri, S. Moriguti, and Y. Takasawa, "On a certain quadrature formula," J. Comput. Appl. Math., no. 17, pp. 3-20, 1987.

[8] S. M. Rao, D. R. Wilton, and A. W. Glisson, "Electromagnetic scattering by surfaces of arbitrary shape," IEEE Trans. Antennas Propag., vol. 30, no. 5, pp. 409-418, May 1982.

[9] S. López-Peña, A. G. Polimeridis, and J. R. Mosig, "On the analyticnumeric treatment of weakly singular integrals on arbitrary polygonal domains," Prog. Electromagn. Res. PIER, vol. 117, pp. 339-355, 2011.

[10] M. Mori, "Quadrature formulas obtained by variable transformation and the DE-rule," J. Comput. Appl. Math., no. 112, pp. 119-130, 1985.

[11] D. H. Bailey, K. Jeyabalan, and X. S. Li, "A comparison of three highprecision quadrature schemes," Experimental Mathematics, vol. 3, no. 14, pp. 317-329, 2005.

[12] A. G. Polimeridis and J. R. Mosig, "Complete semi-analytical treatment of weakly singular integrals on planar triangles via the direct evaluation method," Int. J. Numerical Methods Eng., vol. 83, pp. 1625-1650, 2010.

[13] J. S. Asvestas, S. P. Yankovich, and O. E. Allen, "Calculation of the impedance matrix inner integral to prescribed accuracy," IEEE Trans. Antennas Propag., vol. 58, no. 2, pp. 479-487, Feb. 2010. 